\title{
Predicting Time to Nursing Home Care and Death in Individuals With Alzheimer Disease
}

\author{
Yaakov Stern, PhD; Min-Xing Tang, PhD; Marilyn S. Albert, PhD; Jason Brandt, PhD; Diane M. Jacobs, PhD; \\ Karen Bell, MD; Karen Marder, MD; Mary Sano, PhD; Devangere Devanand, MD; \\ Steven M. Albert, PhD; Frederick Bylsma, PhD; Wei-Yann Tsai, PhD
}

Objective.-To develop and validate an approach that uses clinical features that can be determined in a standard patient visit to estimate the length of time before an individual patient with Alzheimer disease $(A D)$ requires care equivalent to nursing home placement or dies.

Design.-Prospective cohort study of 236 patients, followed up semiannually for up to 7 years. A second validation cohort of 105 patients was also followed.

Setting.-Three AD research centers.

Patients.-All patients met National Institute of Neurological and Communicative Disorders and Stroke-Alzheimer's Disease and Related Disorders Association (NINCDS-ADRDA) criteria for probable AD and had mild dementia at the initial visit.

Intervention.-Predictive features, ascertained at the initial visit, were sex, duration of illness, age at onset, modified Mini-Mental State Examination (mMMS) score, and the presence or absence of extrapyramidal signs or psychotic features.

Main Outcome Measures.-(1) Requiring the equivalent of nursing home placement and (2) death.

Results.-Prediction algorithms were constructed for the 2 outcomes based on Cox proportional hazard models. For each algorithm, a predictor index is calculated based on the status of each predictive feature at the initial visit. A table that specifies the number of months in which $25 \%, 50 \%$, and $75 \%$ of patients with any specific predictor index value are likely to reach the end point is then consulted.Survival curves for time to need for care equivalent to nursing home placement and for time to death derived from the algorithms for selected predictor indexes fell within the $95 \%$ confidence bands of actual survival curves for patients. When the predictor variables from the initial visit for the validation cohort patients were entered into the algorithm, the predicted survival curves for time to death fell within the $95 \%$ confidence bands of actual survival curves for the patients.

Conclusions.-The prediction algorithms are a first but promising step toward providing specific prognoses to patients, families, and practitioners. This approach also has clear implications for the design and interpretation of clinical trials in patients with $A D$.

JAMA. $1997 ; 277: 806-812$

From the Departments of Neurology (Drs Stern, Tang, Jacobs, Bell, Marder, Sano, and S. M. Albert), Psychiatry (Drs Stern and Devanand), and Biostatistics (Dr Tsai), and the Gertrude H. Sergievsky Center and Taub Center for Alzheimer's Disease Research (Drs Stern, Tang, Jacobs, Bell, Marder, Sano, and S. M. Albert), Columbia University College of Physicians and Surgeons, New York, NY; the Department of Psychiatry and Behavioral Sciences, Johns Hopkins University School of Medicine, Baltimore, Md (Drs Brandt and Bylsma): and the Departments of Psychiatry and Neurology. Massachusetts General Hospital, Harvard Medical School, Boston, Mass (Dr M. S. Albert).

Reprints: Yaakov Stern. PhD, Sergievsky Center, 630 W 168th St, New York, NY 10032. are associated with more rapid disease course. ${ }^{1-6}$ This article presents the development and validation of an approach that uses these clinical features to estimate the length of time before a patient requires nursing home care or dies. These algorithms represent the first attempt we know of to make such predictions for individual patients.

\section{METHODS}

Subjects were members of the Predictors Study cohort. ${ }^{7}$ A total of 236 patients with probable $\mathrm{AD}$ were recruited at 3 sites: Columbia University College of Physicians and Surgeons, New York, NY; Johns Hopkins University School of Medicine, Baltimore, Md; and Massachusetts General Hospital, Boston. Inclusion and exclusion criteria, recruitment methods, and the full evaluation battery have been described. ${ }^{7}$ Each patient met $\mathrm{Na}$ tional Institute of Neurological and Communicative Disorders and Stroke-Alzheimer's Disease and Related Disorders Association (NINCDS-ADRDA) criteria for probable $\mathrm{AD},{ }^{8}$ with 1 exception described below. To ensure that severity of dementia was mild at study entry, all patients were required to have a modified Mini-Mental State Examination (mMMS) score of 30 or above (corresponding to approximately 16 on the standard Mini-Mental State Examination [MMSE]). To ensure accurate initial assessment of psychotic symptoms, all patients were required to not be treated with neuroleptic medications for at least 1 month prior to their initial evaluation.

Exclusion criteria were history or current clinical evidence of substance abuse, schizophrenia, schizoaffective disorder, or major affective disorder prior to the onset of intellectual decline; any electroconvulsive therapy treatment within 2 years of recruitment, or 10 or more electroconvulsive treatments at any time; history 
or clinical signs of stroke or a Hachinski Ischemic Score ${ }^{9}$ of 5 or more. Patients with small subcortical lesions on computed tomographic or magnetic resonance imaging scans that were clinically and historically silent and were judged to be less than $2 \mathrm{~cm}$ in diameter were included, as were those with diffuse symmetric periventricular lucencies. However, patients with cortical lesions of any size or location or with focal cortical atrophy in a specific vascular distribution were excluded.

This project was approved by the institutional review board at each participating institution, and all patients provided written informed consent.

\section{Procedures}

Predictors.-At the initial visit, the following predictive features were assessed:

Duration of Illness. - The clinician estimated disease duration based on interviews with the patient and informants. As part of the standard medical history, the clinician asked the informants to date the onset of clinical features such as memory, performance, and language changes in order to date the onset of the disease. Clinicians' estimates were separately confirmed using a standardized interview technique. ${ }^{10}$

Age at Onset.-Based on this interview, age at onset of dementia was characterized as young (younger than 65 years) or old (age 65 years or older).

Extrapyramidal Signs.-Selected items from the Unified Parkinson's Disease Rating Scale (UPDRS) ${ }^{11}$ were used to rate extrapyramidal signs. Hypophonia, masked faces, resting tremor, rigidity (neck and each limb), brady/ hypokinesia, and posture and gait abnormalities were rated as absent, slight, mild to moderate, marked, or severe. ${ }^{12}$ Patients who had at least 1 sign rated as mild to moderate were considered to have extrapyramidal signs since ratings of this severity are more reliable and are apt to be noted by the average clinician..$^{12}$ Our analyses focused on nondrug-induced extrapyramidal signs. If a patient's signs were possibly or probably drug-induced, then they were not considered extrapyramidal signs for the purpose of prediction and were not included in the statistical analyses.

Psychosis.-A semistructured interview, the Columbia University Scale for Psychopathology in Alzheimer's Disease (CUSPAD), ${ }^{13}$ was used to elicit information about delusions, hallucinations, and other specific behavioral signs occurring during the month prior to assessment. Specific delusions queried included paranoid delusions, delusions of abandonment, somatic delusions, and misidentifications. We used the presence of delusions or hallucinations at the initial visit as evidence of psychosis.

Cognition-Cognitive function was examined using the mMMS, ${ }^{14,15}$ which includes the items from the standard MMSE, ${ }^{16}$ the Wechsler Adult Intelligence Scale-Revised (WAISR) Digit Span subtest, ${ }^{17}$ and additional attention/ calculation, general knowledge, language, and construction items. The maximum score on the mMMS is 57 .

Other measures were also obtained at each study visit, including the Blessed Dementia Rating Scale (BDRS) (Part 1) to assess functional capacity, and the Clinical Dementia Rating (CDR) Scale ${ }^{19}$ to rate the overall severity of the dementia.

Outcomes.-Patients were followed up semiannually for up to 7 years. The following 2 outcomes were considered.

Health-Related Facility Care.-The "equivalent institutional care"20 that the patient was receiving was rated at each 6-month follow-up interval. This rating summarizes the interviewer's impression, based on data from the entire study protocol, ${ }^{7}$ of the care the patient received and required, regardless of the patient's location. Categories are limited home care; adult home (a supervised setting with regular assistance in most activities); and health-related facility (nursing home care). We used the rating equivalent to care in a health facility as an end point for prediction since it is equivalent to nursing home placement, an important event for families of patients with $\mathrm{AD}$. Interrater reliability for the equivalent care rating is good; the intraclass correlation coefficient was 0.73 in a study of 20 patients. ${ }^{20}$

Death-Autopsies were obtained whenever possible. When autopsies were not obtained, we typically learned of patients' deaths from family members or when attempting to complete follow-up visits. For patients who could not be contacted for follow-up or were otherwise unavailable for follow-up, death information was obtained as available through the National Death Index.

Validation Population.-A population was selected in which to assess the accuracy of the algorithm with respect to the death outcome. We used data from elders with dementia in North Manhattan who are being followed up prospectively as part of another epidemiologic study. None of these patients were members of the Predictors Study cohort. These patients also met NINCDSADRDA criteria for probable $A D$ based on neurological examination, neuropsychological testing, and functional evaluation. Duration of illness, extrapyramidal signs, and mortality information were collected as described above. The mMMS was not administered to this population, so we developed a regression equation to estimate mMMS scores on the basis of 2 tests they did receive, the short Blessed test ${ }^{21}$ and total recall on the Selective Reminding Test. ${ }^{22}$ The equation was based on a group of 255 probable AD patients who took all 3 tests. The multiple $R$ for the regression equation was 0.72 . Patients were included in the validation analysis if the UPDRS, duration of illness estimates, and data required to estimate the mMMS score were available and if the estimated mMMS was at least 25 . This provided 105 patients for the current analyses.

Apolipoprotein E.-Apolipoprotein E $(A P O E$ ) studies were not an original component of this study. However, we wanted to determine whether $A P O E$ genotype influenced the prediction algorithms that we developed. Beginning in the sixth year of the study, available subjects were approached to contribute blood samples for analysis. In addition, analyses were conducted on frozen brain tissue of 12 patients who had died and contributed their brains for postmortem studies.

The patterm of each subject's APOE isoforms was determined using the method of Hixson and Vernier. ${ }^{23}$ The white blood cells were isolated from fresh blood samples after centrifugation at 2000 rpm for 20 minutes at $4^{\circ} \mathrm{C}$, and the DNA was digested with $H h$ AI. Leukocyte DNA was amplified by polymerase chain reaction (PCR) using specifically synthesized oligonucleotide primers and Taq polymerase. The amplified $A P O E$ products were then digested with 5 units of $\mathrm{HaAI}$ enzyme at $37^{\circ} \mathrm{C}$ for 4 hours. The digest was electrophoresed on a $12 \%$ nondenaturing polyacrylamide gel for 3 hours at a constant current of $10 \mathrm{~mA}$. The gels were then treated with ethidium bromide for 10 to 15 minutes, and the DNA fragments were visualized by ultraviolet illumination. Fragments of DNA of known size were used as markers.

\section{Data Analyses}

Cox Analysis. - Cox proportional hazard analyses used the predictors listed above: extrapyramidal signs, psychosis, and age at onset. Initial mMMS score, estimated duration of illness, and sex were also considered as potential covariates. Since older patients might reach the defined end points sooner, all Cox analyses were stratified by the median age at first visit, 73 years.

Reaching the equivalent of nursing home care and death were the 2 outcomes considered. For each outcome, we calculated a Cox proportional hazards model that used months from the initial visit as the timing variable. After calculating each model, it was "trimmed back" to include only those covariates that were associ- 
Table 1.-Cox Proportional Hazard Analysis Predicting Reaching an Institutional Care Rating Equivalent to Health-Related Facility Care*

\begin{tabular}{lcc}
\hline \multicolumn{1}{c}{ Predictor } & $\boldsymbol{\beta}$ & $\boldsymbol{R R}(95 \% \mathrm{Cl})$ \\
\hline $\begin{array}{l}\text { EPS present } \\
\text { Psychotic symptoms }\end{array}$ & 0.9419 & $2.56(1.49-4.41)$ \\
$\quad \begin{array}{c}\text { present } \\
\text { Young age at onset, }\end{array}$ & 0.4027 & $1.50(1.04-2.15)$ \\
$\quad<65$ y & 0.4848 & $1.62(0.99-2.67)$ \\
$\begin{array}{c}\text { mMMS score } \\
\quad \text { (each unit) }\end{array}$ & -0.0724 & $0.93(0.90-0.96)$ \\
Duration of illness, y & -0.0617 & $0.94(0.87-1.01)$ \\
\end{tabular}

*RR indicates relative risk; $\mathrm{Cl}$, confidence interval EPS, extrapyramidal signs; and mMMS, modified MiniMental State Examination.

ated with significant or borderline $(P<.10)$ significant increases in relative risk. We used the martingale methods to check the proportional hazards assumption. ${ }^{24}$

Derivation of the Predictor Algorithms.-Predictor algorithms for nursing home care and mortality endpoints were then developed, based on the Cox models. Using a Cox model, a survival curve for any individual patient can be estimated based on the value of that patient's covariates.

The algorithm has 2 steps. First, a patient's predictor index is calculated. In Cox models, the risk associated with a given set of covariates can be specified by summing the products of the covariates and their associated $\beta$ weights.

Second, a table that specifies the number of months in which a patient with a specific predictor index value is likely to reach the end point of interest is consulted. To construct the table, we calculated the predictor index for each patient in the cohort and then estimated the survival curve for a representative range of values. Based on these curves we determined and entered into the table the number of months in which $25 \%, 50 \%$, and $75 \%$ of patients with a specific index value would be expected to reach an endpoint. Since the Cox analyses were stratified at age 73 years, separate tables were constructed for the young and old age groups.

Validation of the Predictor Algorithms.-We compared the algorithmderived survival curve for a specific predictor index value to a survival curve describing patients who had similar predictor indexes at their initial visit. We posited that if these curves are similar, then the predictions based on the index would closely match the actual survival distribution of patients with that index. To compare these 2 curves, we calculated the $95 \%$ confidence band around the survival curve for actual patient data and then determined whether the curve derived from the prediction algorithm was contained within this band.

The initial validation was performed in the same cohort used to derive the algorithm to test how well the derived Cox

Table 2.-Guide for Calculating a Patient's Predictor Index for Health-Related Facility Care*

\begin{tabular}{|c|c|c|c|c|c|}
\hline Predictor & Coding & Value & & Weight & Value $\times$ Weight \\
\hline EPS & 0 Absent, 1 present & & $x$ & -0.9419 & \\
\hline Psychotic symptoms & 0 Absent, 1 present & & $x$ & -0.4027 & \\
\hline Age at onset & 0 Normal, 1 young $(<65 \mathrm{y})$ & & $x$ & -0.4848 & \\
\hline mMMS & Actual score & & $x$ & 0.0724 & \\
\hline Duration of illness & Estimated years & & $x$ & 0.0617 & \\
\hline
\end{tabular}

*Using the coding guidelines, a value for each predictor is entered into the "Value" column. The product of each predictor's value and weight is entered in the far right column. The predictor index is the sum of these products. Using this predictor index, estimates of the time until health-related facility (HRF) care will be required can be looked up in Table 3. EPS indicates extrapyramidal signs; and mMMS, modified Mini-Mental State Examination.

Table 3.-Specific Prediction of Months Until a Patient Will Require Health-Related Facility Care, Based on Predictor Index*

\begin{tabular}{|c|c|c|c|c|c|c|}
\hline \multirow[b]{2}{*}{$\begin{array}{l}\text { HRF Care } \\
\text { index }\end{array}$} & \multicolumn{3}{|c|}{ Young ( $\leq 73 y$ ) } & \multicolumn{3}{|c|}{ Old $(>73 y)$} \\
\hline & $25 \%$ & $50 \%$ & $75 \%$ & $25 \%$ & $50 \%$ & $75 \%$ \\
\hline 1.0 & 12.2 & 16.9 & 24.6 & 8.9 & 13.3 & 18.8 \\
\hline 1.2 & 13.1 & 18.9 & 29.1 & 11.1 & 15.7 &, 19.5 \\
\hline 1.4 & 13.1 & 21.2 & 30.3 & 11.5 & 16.8 & 23.6 \\
\hline 1.6 & 14.9 & 24.3 & 36.0 & 12.0 & 18.4 & 24.1 \\
\hline 1.8 & 16.8 & 25.9 & 37.6 & 12.7 & 18.9 & 28.3 \\
\hline 2.0 & 18.3 & 29.7 & 42.6 & 13.4 & 22.9 & 30.3 \\
\hline 2.2 & 20.2 & 34.0 & 27.8 & 16.3 & 23.7 & 37.7 \\
\hline 2.4 & 22.7 & 36.8 & 53.0 & 17.9 & 24.7 & 42.7 \\
\hline 2.6 & 24.7 & 41.4 & 54.9 & 18.9 & 28.8 & 47.2 \\
\hline 2.8 & 29.2 & 43.9 & 59.4 & 20.7 & 34.1 & 61.4 \\
\hline 3.0 & 31.1 & 49.9 & 60.7 & 23.6 & 38.1 & 66.6 \\
\hline 3.2 & 36.3 & 53.5 & 60.7 & 24.5 & 44.4 & \\
\hline 3.4 & 38.2 & 58.6 & & 28.8 & 48.6 & \\
\hline 3.6 & 42.6 & 60.7 & & 30.4 & 66.2 & \\
\hline 3.8 & 47.8 & 67.1 & & 38.0 & & \\
\hline 4.0 & 53.0 & & & 44.3 & & \\
\hline 4.2 & 54.9 & & & 47.6 & & \\
\hline 4.4 & 59.4 & & & 61.4 & & \\
\hline
\end{tabular}

*For each predictor index value (as calculated in Table 2), the table shows number of months within which the specified percentage of comparable patients require the equivalent of health-related facility (HRF) care. Values for younger ( $\leq 73$ years) and older (>73 years) patients are provided separately. Empty cells represent patients with milder severity at their initial visit (and thus higher predictor index values) for whom there are insufficient data to generate predictions.

models and their implementation in the predictor algorithm fit the actual patient data. To do this, we calculated KaplanMeier curves and 95\% confidence bands for patients whose predictor indexes were within a 1-unit range. These curves were compared with those generated by the algorithm for a predictor index in the center of that range. For example, we calculated the survival curve for patients whose nursing home predictor indexes ranged from 1.5 to 2.5 and compared this curve with the curve generated by the algorithm for an index of 2.0. The selection of predictor index ranges for these analyses was guided by the distribution of the index scores in each case. Since our initial Cox analyses were stratified by age, these validation analyses were conducted separately for patients in the 2 age groups.

We next cross-validated the algorithm for mortality in the sample of North Manhattan residents. Based on the baseline visit data, we calculated each patient's predictor index for death. We then calculated a survival curve with
95\% confidence band for the patients and compared it with the survival curve associated with the median predictor index in 2 ranges of predictor values.

\section{RESULTS}

Mean (SD) age of the 236 patients at intake into the study was 73.1 (8.9) years. Mean estimated duration of illness was 3.9 (2.4) years. There were 96 men and 140 women; $84 \%$ of the group were nonHispanic whites. Mean mMMS at intake was 37.9 (5.6), mean BDRS was 8.0 (3.5), and mean CDR was $1.1(0.3)$, all consistent with mild disease severity. Medical conditions and medications at intake have been summarized previously. ${ }^{\top}$ Of the 236 patients, $26 \%$ were taking antihypertensive medications; $9 \%$, antidepressants; $6 \%$, sedative hypnotics; $3 \%$, thyroid medication; and $6 \%$ of patients were taking more than 1 of these medications. $\mathrm{Ab}$ normal findings on medical evaluation were identified in $28 \%$, but none of these was felt to contribute to the presentation of dementia. 
At baseline, 26 patients (11\%) had at least 1 extrapyramidal sign that was rated in the mild to moderate range and was not drug induced; 15 patients had extrapyramidal signs that were or might have been drug induced and were excluded from the analyses; and 103 patients had at least 1 psychotic feature. Age at onset was considered young ( $<65$ years) in 74 patients. At the time of these analyses, 220 patients were followed up for 1 or more years, 199 for 2 or more years, 164 for 3 or more years, 138 for 4 or more years, 94 for 5 or more years, and 43 for 6 or more years. Maximum follow-up time at this point is 7 years.

\section{Health Related Facility Care}

Over the follow-up period, 164 patients were judged to have reached the point where they received the equivalent of health related facility care. Of these, 63 were actually admitted to a nursing home at the same time and another 34 were admitted sometime later. No subject was admitted to a nursing home without being judged as requiring that care. Using $t$ tests, we compared the scores on several indexes of dementia severity among patients who were and were not admitted to a nursing home at the point that they were first rated as requiring the equivalent of nursing home care. The 2 groups did not differ significantly in level of cognitive dysfunction as assessed by the mMMS (mean mMMS $=20.3$ [11.6] and 22.3 [13.9], respectively); functional disability as assessed by total score on the BDRS (mean BDRS $=15.0$ [3.8] and 15.1 [5.3], respectively); basic activities of daily living as assessed by the BDRS (mean score $=3.2$ [2.2] and 3.5 [3.1], respectively); and overall dementia severity as rated by the CDR (mean $\mathrm{CDR}=2.1$ [0.7] and 2.2 [0.9], respectively).

Results of the Cox analysis are summarized in Table 1. The relative risk ( $R R$ ) of reaching this endpoint was significantly increased in patients who had extrapyramidal signs or psychotic symptoms at their initial visit and in patients with young age at disease onset. Higher mMMS scores were associated with significantly reduced risk of reaching the endpoint, and a similar trend was noted for longer estimated disease duration at the initial visit. The sex of the patient was not a significant predictor of nursing home level care.

In order to check the assumptions of the Cox regression model, we plotted the martingale residuals against months of follow-up. ${ }^{24}$ All but 4 subjects fell within the predicted horizontal band around 0 , satisfying the assumptions of proportional hazards. In supplementary analysis, we compared the Cox models calculated for each separately. Results were compatible across the 3 sites.
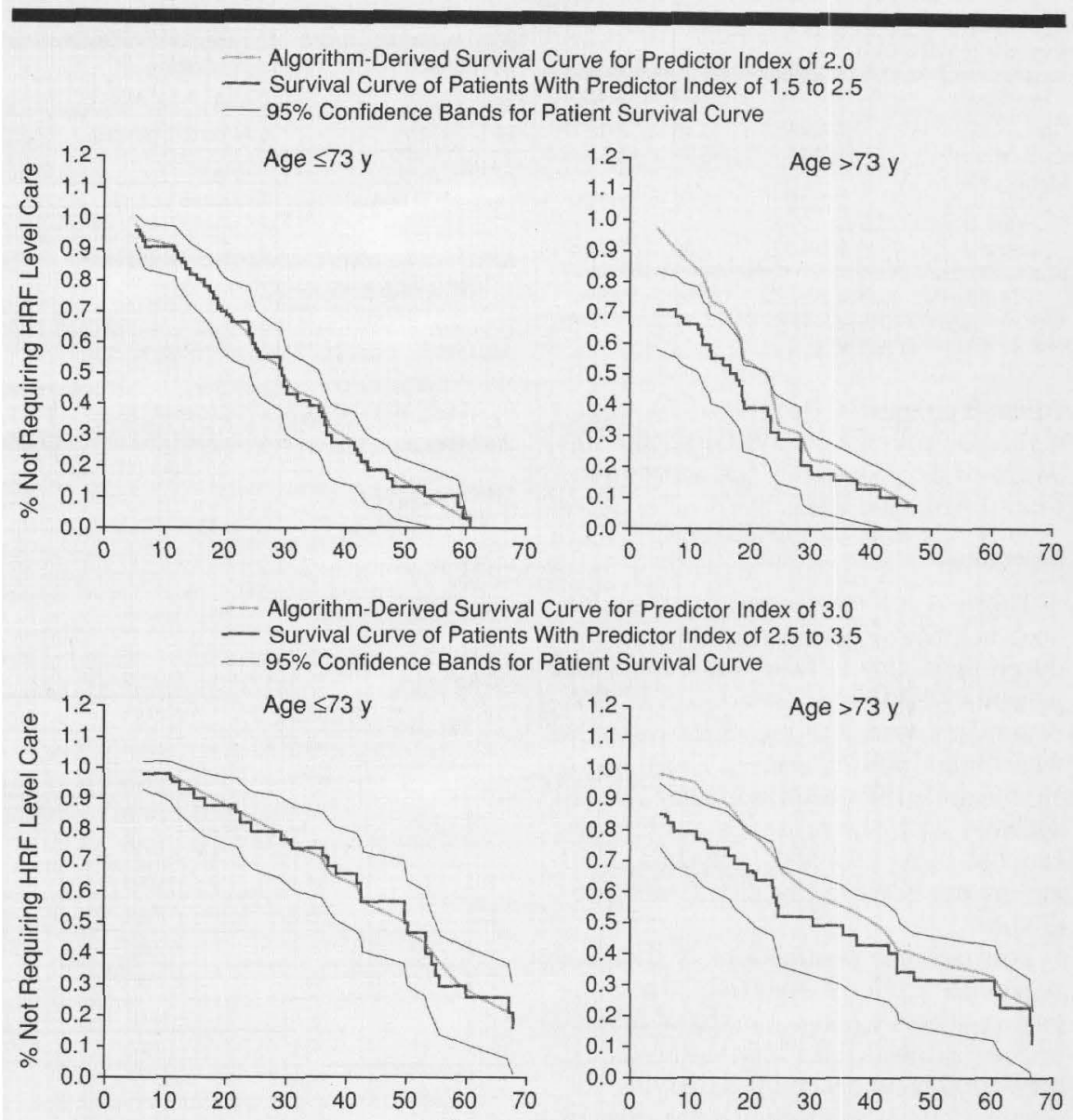

- Algorithm-Derived Survival Curve for Predictor Index of 4.0

- Survival Curve of Patients With Predictor Index of 3.5 to 4.5

- $95 \%$ Confidence Bands for Patient Survival Curve
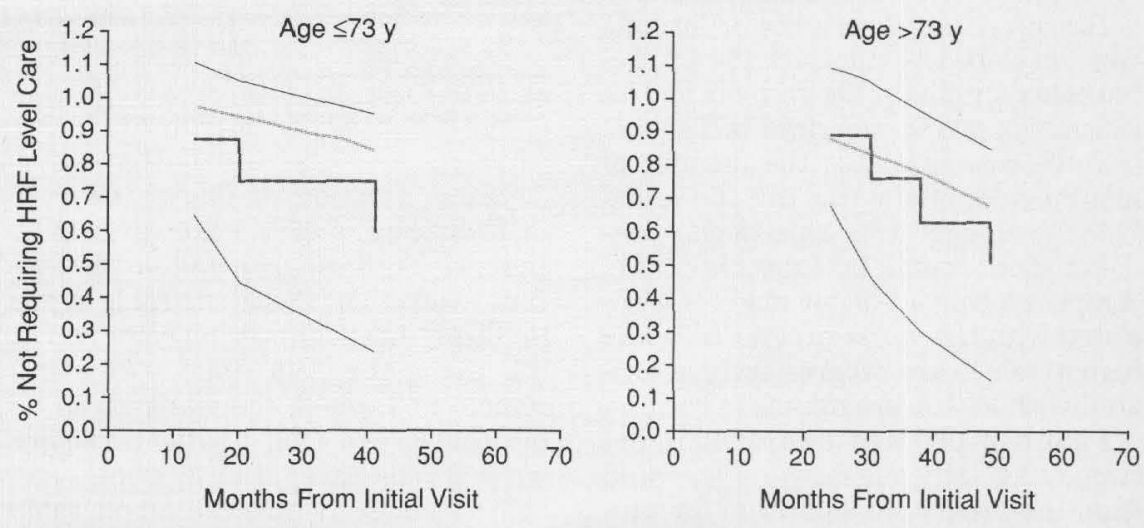

Figure 1.-Time to need for care equivalent to placement in health-related facility (HRF), comparing algorithm-derived survival curves with survival curves for patients aged 73 years or younger and patients older than 73 years at initial visit. Nearly all patient data points fall within $95 \%$ confidence bands, indicating that algorithms are valid models for actual patients with the given predictor index.

Based on the $\beta$ weights, a formula was developed to calculate the predictor index for nursing home level care. The formula and its calculation are summarized in Table 2.

Table 3 summarizes the number of months from intake that $25 \%, 50 \%$, and $75 \%$ of subjects with a particular predictor index would be expected to require nursing home care. Note that a lower predictor index is associated with the worst prognoses, while higher values are progressively associated with better prognoses.

Figure 1 displays the comparisons between the survival curves generated from the prediction algorithm for predictor indexes of 2,3 , and 4 and survival curves of patients whose index at their initial visit was within 0.5 unit of this 
Table 4.-Cox Proportional Hazard Analysis for Predicting Mortality*

\begin{tabular}{crc}
\hline Predictor & $\boldsymbol{\beta}$ & RR $(95 \%$ Cl) \\
\hline Female sex & -0.7046 & $0.49(0.32-0.77)$ \\
EPS present & 1.2825 & $3.61(2.12-6.14)$ \\
mMMS score & -0.0310 & $0.97(0.93-1.00$ \\
$\quad$ (each unit) & -0.1052 & $0.90(0.83-0.98)$ \\
$\begin{array}{c}\text { Duration of } \\
\text { illness, } y\end{array}$ & - &
\end{tabular}

${ }^{*} \mathrm{RR}$ indicates relative risk; $\mathrm{Cl}$, confidence interval; EPS, extrapyramidal signs; and mMMS, modified MiniMental State Examination.

value. The curves generated from the algorithms almost always fall within the $95 \%$ confidence bands of the curves generated from the data.

\section{Mortality}

There were 99 deaths. In the Cox analyses there was a significantly reduced mortality RR for women and for patients with longer duration of illness. A similar trend was noted for patients with higher mMMS scores. There was a significantly increased mortality $R R$ associated with the presence of extrapyramidal signs (Table 4). Psychosis and age at onset were not significant predictors.

To check the assumptions of the Cox regression model, we plotted the martingale residuals against months of followup. ${ }^{24}$ All subjects fell within the predicted horizontal band around 0 , satisfying the assumptions of proportional hazards. In supplementary analysis, we compared the Cox models calculated for each separately. Results were compatible across the 3 sites.

Based on the $\beta$ weights, a formula was developed to calculate the predictor index for death. The formula and its calculation are summarized in Table 5 .

Table 6 summarizes the number of months from intake that $25 \%, 50 \%$, and $75 \%$ of subjects with a particular predictor index would be expected to die. Again, a lower predictor index is associated with the worst prognoses, while higher values are progressively associated with better prognoses.

Figure 2 displays comparisons between the survival curves generated from the prediction algorithm for predictor indexes of 1 and 2 and survival curves of patients whose index was within 0.5 unit of these values at their initial visit. The curves generated from the algorithms fall well within the $95 \%$ confidence bands of the curves generated from the data.

\section{Applying the Algorithms}

To illustrate the application of the prediction algorithms for nursing home care and death to a particular patient, we provide the following example. A 76year-old woman with an estimated age at onset of 73 years and an estimated

Table 5.-Guide for Calculating a Patient's Predictor Index for Death*

\begin{tabular}{|c|c|c|c|c|c|}
\hline Predictor & Coding & Value & & Weight & Value $\times$ Weight \\
\hline Sex & 0 Male, 1 female & & $x$ & 0.7046 & \\
\hline EPS & 0 Absent, 1 present & & $x$ & -1.2825 & \\
\hline mMMS & Actual score & & $x$ & 0.0310 & \\
\hline Duration of illness & Estimated years & & $x$ & 0.1052 & \\
\hline
\end{tabular}

"Using the coding guidelines, a value for each predictor is entered into the "Value" column. The product of each predictor's value and weight is entered in the far right column. The predictor index is the sum of these products. Using this predictor index, estimates of the time until death can be looked up in Table 6. EPS indicates extrapyramidal signs; and mMMS, modified Mini-Mental State Examination.

Table 6.-Specific Mortality Predictions for a Patient, Based on Predictor Index*

\begin{tabular}{|c|c|c|c|c|c|c|}
\hline \multirow{2}{*}{$\begin{array}{l}\text { Death } \\
\text { Index }\end{array}$} & \multicolumn{3}{|c|}{ Young ( $\leq 73$ y) } & \multicolumn{3}{|c|}{ Old $(>73 y)$} \\
\hline & $25 \%$ & $50 \%$ & $75 \%$ & $25 \%$ & $50 \%$ & $75 \%$ \\
\hline 0 & 18.9 & 33.6 & 44.1 & 13.4 & 20.8 & 37.9 \\
\hline 0.2 & 19.0 & 36.4 & 47.3 & 16.2 & 21.6 & 40.5 \\
\hline 0.4 & 21.8 & 40.1 & 54.1 & 16.7 & 23.3 & 44.3 \\
\hline 0.6 & 22.0 & 43.4 & 55.5 & 18.8 & 32.2 & 47.5 \\
\hline 0.8 & 33.5 & 45.2 & 55.7 & 20.8 & 39.9 & 51.0 \\
\hline 1.0 & 35.2 & 50.4 & 58.6 & 21.9 & 43.6 & 57.2 \\
\hline 1.2 & 39.3 & 54.9 & 68.6 & 23.3 & 46.3 & 63.1 \\
\hline 1.4 & 41.3 & 55.5 & 71.0 & 26.7 & 50.8 & 64.0 \\
\hline 1.6 & 44.5 & 57.8 & 76.7 & 37.9 & 57.0 & 67.1 \\
\hline 1.8 & 47.3 & 67.0 & 76.7 & 41.3 & 60.1 & 73.4 \\
\hline 2.0 & 54.1 & 69.5 & 77.1 & 44.9 & 63.6 & 74.9 \\
\hline 2.2 & 55.5 & 72.2 & 77.1 & 47.5 & 64.6 & 80.3 \\
\hline 2.4 & 55.7 & 76.7 & 77.5 & 60.0 & 73.4 & 80.3 \\
\hline 2.6 & 60.7 & 76.7 & & 59.0 & 74.9 & \\
\hline 2.8 & 68.6 & 77.1 & & 63.1 & 80.3 & \\
\hline 3.0 & 70.1 & 77.5 & & 64.3 & 80.3 & \\
\hline 3.2 & 76.7 & & & 67.1 & & \\
\hline 3.4 & 76.7 & & & 73.4 & & \\
\hline 3.6 & 77.1 & & & 74.9 & & \\
\hline 3.8 & 77.1 & & & 80.3 & & \\
\hline 4.0 & 77.5 & & & & & \\
\hline
\end{tabular}

*For each predictor index value (as calculated in Table 5), the table shows number of months within which the specified percentage of comparable patients die. Values for younger ( $\leq 73$ years) and older ( $>73$ years) patients are provided separately. Empty cells represent patients with milder severity at their initial visit (and thus higher predictor index values) for whom there are insufficient data to generate predictions.

3 years' duration of illness. Current mMMS score is 45; no extrapyramidal signs or psychosis is noted at the current evaluation. Based on Table 2, the predictor index for nursing home care is 3.4. Using the "Old" (age $>73$ years) column in Table 3 , this patient can be told that $25 \%$ of similar patients require nursing home care within 29 months and $50 \%$ within 49 months. Based on Table 5 , the predictor index for death is 2.4 . Using the "Old" (age >73 years) column in Table 6 , this patient can be told that $25 \%$ of similar patients die within 60 months, $50 \%$ within 73 months, and $75 \%$ within 80 months.

\section{Validation Population}

Mean age at first visit of the validation population was $82.5(5.9)$ years. Mean estimated duration of illness was $3.4(6.8)$ years. There were 15 men and 90 women. Mean estimated mMMS at intake was 31.8 (4.5). At their initial visit, 30 patients had at least 1 extrapyramidal sign that was rated in the mild to moderate range and was not drug induced. There were 33 deaths.

All subjects were aged 73 years or older at the initial visit, so prediction of time to death was derived from the "Old" column on Table 6 . Figure 3 presents the survival curve and its $95 \%$ confidence band for the subjects' 2 index ranges, along with the survival curve generated from the predictor model for the predictor index at the median of each range. In both cases, the predicted survival falls well within the $95 \%$ confidence bands of the actual survival curves.

\section{Apolipoprotein E}

The APOE genotypes were obtained for 101 of the subjects from a pool of approximately 150 for whom they were potentially available. For the purpose of analysis, patients were dichotomized into 2 groups, those with $(n=58)$ and those without $(n=43)$ at least $1 \in 4$ : allele. The stratified $A P O E$ genotype variable was 
then introduced into the Cox models that were used to generate the prediction algorithms.

In the Cox model, having at least $1 \in 4$ allele was associated with an RR of 0.69 for reaching the nursing home care outcome (95\% confidence interval [CI], 0.381.26). The RRs associated with the other predictors remained comparable to those in the original model. Having at least $1 \in 4$ allele was associated with mortality $\mathrm{RR}$ of 0.56 (95\% CI, 0.27-1.6). The RRs associated with the other predictors remained comparable to those in the original model.

\section{Postmortem Diagnosis}

Autopsies were obtained for 44 of the 99 patients who died. Of these 44 patients, 37 had AD, 4 had senile changes of the Alzheimer type (1 with concomitant progressive subcortical gliosis), 1 had diffuse Lewy body disease, 1 had hippocampal sclerosis, and 1 had a slowly progressive form of Creutzfeldt-Jakob disease.

\section{COMMENT}

Prognosis is a standard part of a medical evaluation, and it is possible to predict outcomes for many medical conditions. However, these predictions have not been available for patients with $\mathrm{AD}$. As with any other disease, prediction rests on the identification of specific disease features that impart prognostic information. We used several clinical features that have been reliably associated with disease course ${ }^{1}$ and are readily ascertainable in a clinical setting to develop predictor algorithms for $\mathrm{AD}$.

The algorithms described here apply best to patients similar to those included in our study. The study was derived from patients seen at specialized $\mathrm{AD}$ centers, who met research criteria for $\mathrm{PAD}$ with mild to moderate disease severity and were ambulatory. Patients with more complex medical histories and specific systemic illnesses may not fit the predictor model in the same way. We restricted the algorithms to the range of predictor indexes actually seen in our cohort at their initial visit.

We validated and cross-validated the accuracy of the algorithms' predictions, but they will benefit from further development and validation. Replication and confirmation of the algorithms in other populations will increase accuracy. Still, this approach can differentiate patients with better and worse prognoses to a degree that is not currently possible.

One encouraging feature of the crossvalidation analysis was that an estimated mMMS score derived from a regression analysis was a useful proxy for the actual score in the equation to derive the predictor index. We have derived equations for estimating mMMS scores from

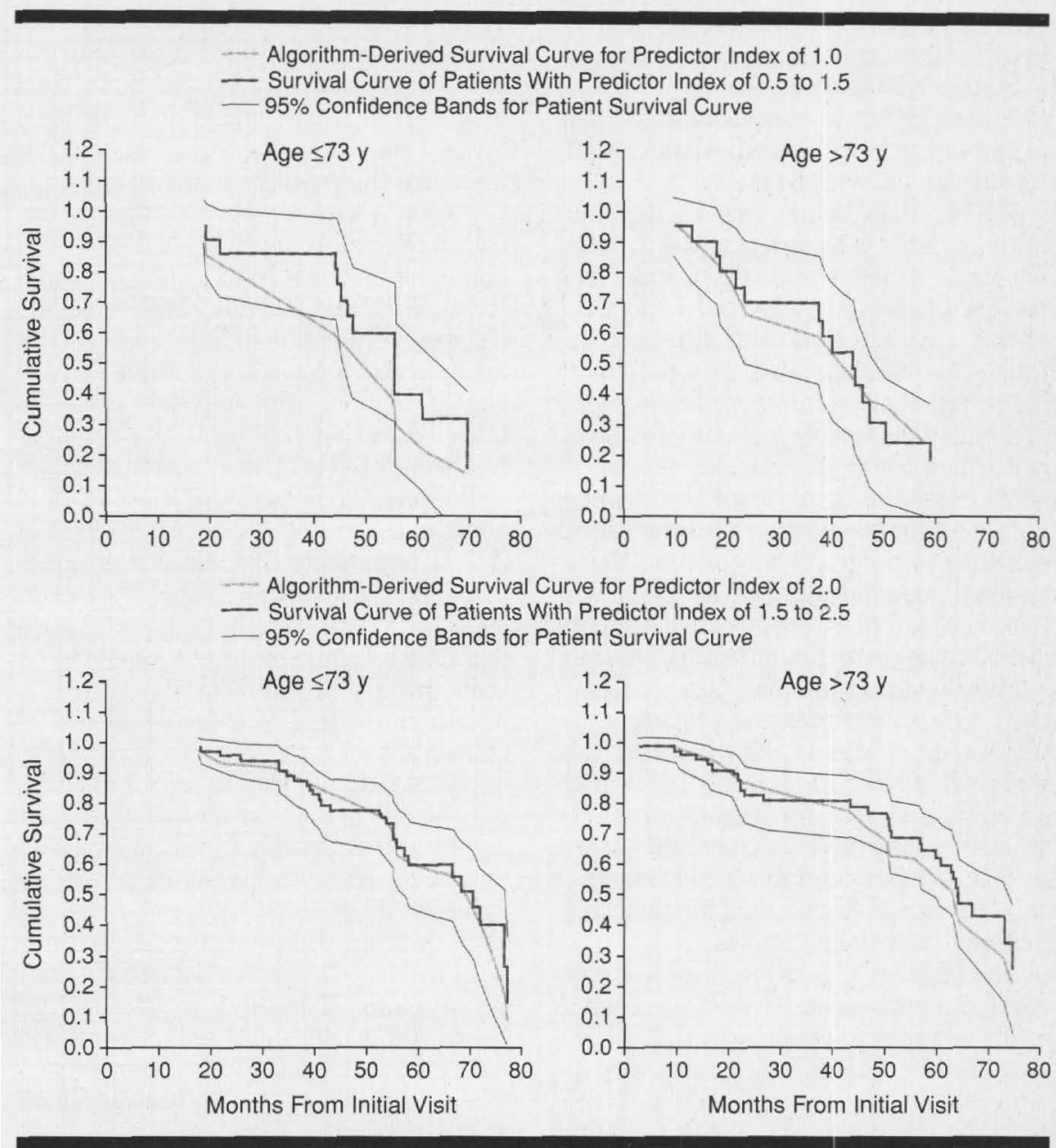

Figure 2.-Time to death, comparing algorithm-derived survival curves with survival curves for patients aged 73 years or younger and patients older than 73 years at initial visit. Nearly all patient data points fall within the $95 \%$ confidence bands, indicating that the algorithms are valid models for actual patients with the given predictor index.

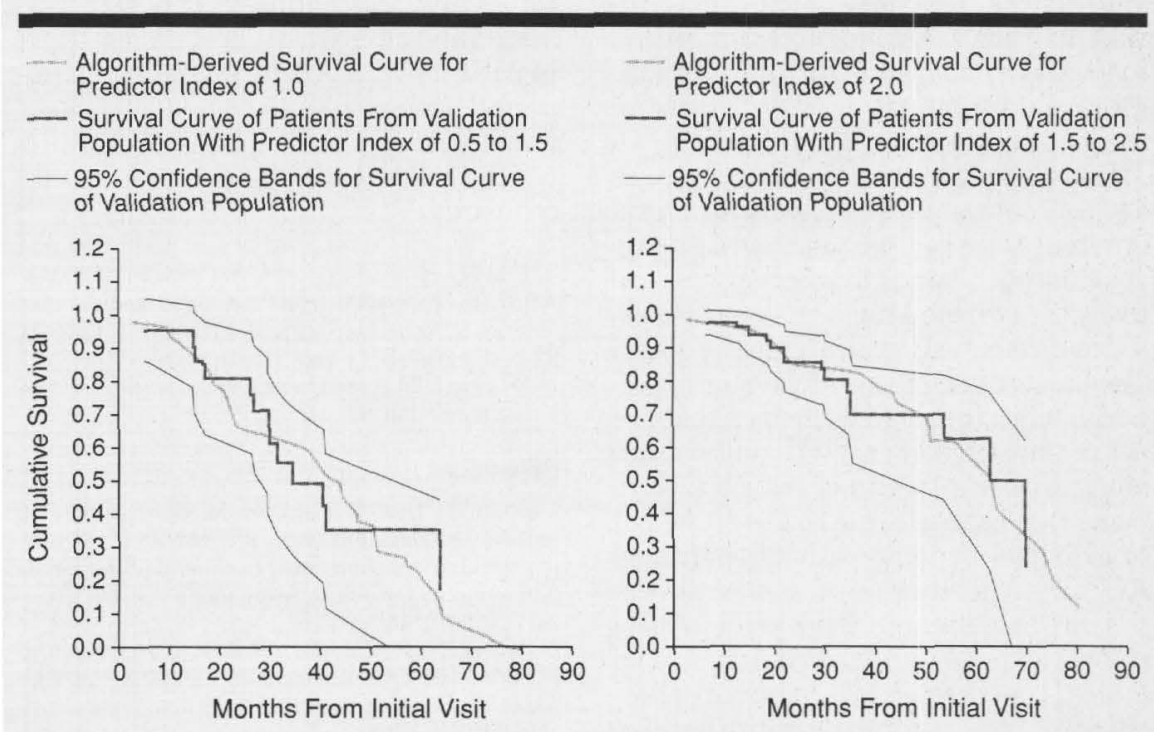

Figure 3.-Time to death, comparing the algorithm-derived survival curve with the survival curve derived from an external validation population of patients with probable Alzheimer disease. All patients in the validation population were older than 73 years. The algorithm-derived survival curve falls within the $95 \%$ confidence bands for validation population, indicating that the algorithm is valid for a new patient population. 
other mental status scale scores including the standard MMSE ${ }^{16}$ (for example, mMMS $=1.73 \mathrm{MMSE}+2.81$ ), and this should broaden the applicability of the predictor algorithms to patients at different clinical centers.

Rather than using actual entry into nursing home care as a study end point, we chose to use the point at which the patient began to receive the equivalent of this care. We chose this approach because the decision to admit a patient to a nursing home is influenced by a range of nonmedical factors including financial, cultural, and familial considerations. In some cases the family may elect to provide the equivalent of nursing home level care in the home. The equivalent institutional care rating that we used was based on the entire range of instruments used in the semiannual evaluations, including several measures of activities of daily living and a measure of patient dependence. ${ }^{20}$ Patients who reached this end point scored comparably on a range of cognitive and functional measures whether or not they were actually placed in a facility. We therefore feel predicting this outcome is more useful for clinicians, patients, and their families.

The current algorithm does not consider the cause of death or the presence of other medical conditions that might contribute differentially to mortality. In adherence with NINCDS-ADRDA criteria for the diagnosis of $\mathrm{AD}$, patients' neurological examinations were notable only for mental status changes but were otherwise unremarkable, and none had medical conditions that would contribute to their dementia. None of the patients had a life threatening illness at study entry. We chose to exclude medical conditions that might arise during follow-up from the algorithm because there is no systematic way to predict their occurrence at the initial visit.

Comparable to the experience in other clinical centers where diagnostic criteria are strictly applied, postmortem data suggest that our diagnostic accuracy was relatively high. Of the 44 autopsies performed, 37 confirmed $\mathrm{AD}$ and another 4 found "senile changes of the Alzheimer type," which indicate $\mathrm{AD}$-like changes that are out of the range of normal aging but are of insufficient severity or distribution to make the diagnosis of AD. We chose not to eliminate patients without confirmed $\mathrm{AD}$ from our analyses for several reasons. First, we acquired autopsies for only half of the patients who died. It would be inconsistent to selectively eliminate cases only from the autopsied group. Second, all of the patients met the standard clinical criteria typically used at AD research centers and were representative of the patients followed up in most $\mathrm{AD}$ cohorts.
Thus, the presented algorithms are derived from and useful for the typical patient diagnosed with AD. Since the final diagnosis is not available at a patient's initial visit, we argue that it should not influence the prediction algorithm.

Patients with a longer estimated duration of symptoms at their initial visit had a better prognosis. Thus, in 2 patients with comparable disease severity, longer duration of illness is actually an indicator of a more benign disease course. This relationship between estimated duration of illness and prognosis has been noted in other diseases as well. ${ }^{25}$

Because the presence of the $A P O E \in 4$ allele is associated with earlier onset of $\mathrm{AD}$, it is possible that disease progression might also be more rapid. However, reports to date have not supported this possibility, ${ }^{26}$ and some have suggested a trend toward slower disease progression in patients with 1 or more $\epsilon 4$ alleles. ${ }^{27,28}$ Our data also suggest that survival was better in patients with at least $1 € 4$ allele, but the $R R$ in the current model was not significant. Although $A P O E$ status may eventually prove to have some predictive value for disease course, we chose to exclude it from the prediction algorithms because the relationship between $A P O E$ genotype and disease progression has not been established, and the algorithms will have greater clinical utility if they rely on clinical signs that are readily ascertained at a patient visit.

We propose these prediction algorithms as a first but promising step toward providing important information about the expected time until disease outcomes to patients, families, and practitioners. In addition to its prognostic value in a clinical setting, this approach may facilitate design and interpretation of clinical trials in patients with AD by providing an expected time course to the equivalent of nursing home placement and death with which the patient's actual course can be compared.

This study was supported by grants AG07370, AG07232, RR00645, from the NIH and by the Charles S. Robertson Gift for Alzheimer's Disease from the Banbury Fund, Huntington, NY.

We thank W. Allen Hauser, MD, for his guidance in implementing this study.

\section{References}

1. Stern Y, Brandt J, Albert M, et al. Utility of extrapyramidal signs and psychosis as predictors of cognitive and functional decline, nursing home admission and death in Alzheimer's disease. Neurology. 1994;44:2300-2307.

2. Jacobs D, Sano M, Marder K, et al. Age at onset of Alzheimer's disease: relation to pattern of $\mathrm{cog}$ nitive dysfunction and rate of decline. Neurology. 1994;44:1215-1220.

3. Stern Y, Mayeux R, Sano M, et al. Predictors of disease course in patients with probable Alzheimer's disease. Neurology. 1987;37:1649-1653.

4. Chui HC, Lyness S, Sobel E, et al. Prognostic implications of symptomatic behaviors in Alzhei- mer's disease. In: Heterogeneity of Alzheimer's Disease. New York, NY: Springer-Verlag; 1992:12-24. 5. Mortimer JA, Ebbitt B, Jun SP, Finch MD. Predictors of cognitive and functional decline in patients with probable Alzheimer's disease. Neurology. 1992;42:1689-1696.

6. Stern Y, Mayeux R, Sano M, Chen J. Prognostic factors for the progression of probable Alzheimer's disease. Bull Clin Neurosci. 1989;54:73-79.

7. Stern Y, Folstein M, Albert M, et al. Multicenter study of predictors of disease course in Alzheimer disease (the 'Predictors Study'), I. Alzheimer Dis Assoc Disord. 1993;7:3-21.

8. McKhann G, Drachman D, Folstein M, et al. Clinical diagnosis of Alzheimer's disease. Neurology. 1984;34:939-944.

9. Mayeux R, Stern Y, Sano M, et al. Clinical and biochemical correlates of bradyphrenia in Parkinson's disease. Neurology. 1987;37:1130-1134. Correction: Neurology. 1987;37:1693.

10. Sano M, Stern Y, Mayeux R, et al. A standardized technique for establishing the onset symptoms of probable Alzheimer's disease. J Clin Exp Neuropsychol. 1987;9:65.

11. Stern MB, Hurting HI. The clinical characteristics of Parkinson's disease and Parkinsonian syndromes: diagnosis and assessment. In: The Comprehensive Management of Parkinson's Disease. New York, NY: PMA Corp; 1978:3-50.

12. Richards $M$, Marder $K$, Bell $K$, et al. Interrater reliability of extrapyramidal signs in a group assessed for dementia. Arch Neurol 1991;48:1147-1149. 13. Devanand DP, Miller L, Richards M, et al. The Columbia University seale for psychopathology in Alzheimer's disease. Arch Neurol 1992:49:371-376. 14. Mayeux R, Stern Y, Rosen J, Leventhal J. Depression, intellectual impairment and Parkinson's disease. Neurology. 1981;31:645-650.

15. Stern Y, Sano M, Paulson J, Mayeux R. Modified Mini-Mental State Examination: validity and reliability. Neurology. 1987;37(suppl 1):179.

16. Folstein MF, Folstein SE, McHugh PR. 'MiniMental State': a practical method for grading the cognitive state of patients for the clinician. I Psychiatr Res. 1975;12:189-198.

17. Wechsler D. Wechsler Adult Intelligence ScaleRevised. New York, NY: Psychological Corp; 1981. 18. Blessed $G$, Tomlinson BE, Roth M. The association between quantitative measures of senile change in the cerebral grey matter of elderly subjects. $\mathrm{Br} J$ Psychol. 1968;114:797-811.

19. Hughes CP, Berg L, Danziger WL, et al. A new clinical scale for the staging of dementia. $\mathrm{Br} J \mathrm{Psy}$ chiatry. 1982;146:566-572.

20. Stern Y, Albert SM, Sano M, et al. Assessing patient dependence in Alzheimer's disease. $J$ Ger. ontol. 1994;49:M216-M222.

21. Katzman R, Brown T, Fuld P, et al. Validation of a short orientation-memory-concentration test of cognitive impairment. Am J Psychiatry. 1983; 140:734-739.

22. Buschke H, Fuld PA. Evaluating storage, retention, and retrieval in disordered memory and learning. Neurology. 1974;24:1019-1025.

23. Hixson J, Vernier D. Restriction isotyping of human apolipoprotein $\mathrm{E}$ by gene amplification and cleavage with HhAI. J Lipid Res. 1991;31:545-548. 24. Collet D. Modelling Survival Data in Medical Research. London, England: Chapman and Hall; 1994:153.

25. Tysnes OB, Vollset SE, Larsen JP, Aarli JA. Prognostic factors and survival in anyotrophic lateral sclerosis. Neuroepidemiology. 1994;13:226-235. 26. Corder EH, Saunders AM, Strittmatter WJ, et al. Apolipoprotein E, survival in Alzheimer's disease patients, and competing risks of death and Alzheimer's disease. Neurology. 1995;45:1323-1328. 27. Frisoni GB, Govoni S, Geroldi C, et al. Gene dose of the $\epsilon 4$ allele of apolipoprotein $\mathrm{E}$ and disease progression in sporadic late-onset Alzheimer's disease. Arn Neurol. 1995;37:596-604.

28. van Dujin CM, de Knijff $P$, Wehnert A, et al. The apolipoprotein $\mathrm{E} \in 2$ allele is associated with an increased risk of early-onset Alzheimer's disease and a reduced survival. Ann Neurol. 1995;37:605-610. 